\title{
An anonymous authenticated key-agreement scheme for multi-server infrastructure
}

\author{
Muhammad Arslan Akram', Zahid Ghaffar ${ }^{1}$, Khalid Mahmood', Saru Kumari ${ }^{2}$, Kadambri Agarwal ${ }^{3}$ \\ and Chien-Ming Chen ${ }^{4^{*}}$
}

*Correspondence:

chienmingchen@ieee.org

${ }^{4}$ College of Computer

Science and Engineering,

Shandong University

of Science and Technology,

Qingdao 266590, China

Full list of author information

is available at the end of the

article

\begin{abstract}
Due to single-time registration, the multi-server authentication provides benefit for getting services from different servers through trusted agent. Generally, users feel hesitation for registering themselves individually with all service providers due to the problem of memorizing the multiple passwords. The multi-server authentication allows a quick access to services by real-time customer validation on public channel. Thereafter, hundreds of multi-server authentication protocols have been introduced. However, the more efficient and robust authentication schemes are being explored by the research academia. We introduce an anonymous scheme that resists the major security threats like impersonation attack, insider attack and password modification attacks in viable computing cost. We use random oracle model for formal security analysis of the proposed scheme. The performance analysis shows that the proposed scheme incurs less computation, energy, communication and storage cost as compared to related protocols. This analysis and comparison show that our proposed scheme is quite effective for the purpose of anonymous authentication and key agreement.
\end{abstract}

Keywords: Multi-server, User anonymity, Authentication, Impersonation, Keyagreement protocol, Internet of Things, Cloud computing

\section{Introduction}

Multi-server authentication (MSA) allows the abrupt access to various online services as compared to single registration, in peer-to-peer environment. $M S A$ architecture [1, 2] is suitable for both sides, i.e. customers and service providers. Because, due to one-time registration with registration center $(R C)$, the customer does not need for remembering multiple passwords. Consequently, the MSA architecture facilitates the service providers to maintain verifier database for each authentic user, in order to avoid multiple registration. To get perk from these services from various servers, the customers rely on a single time registration with $R C$. The $M S A$ environment involves various servers $\left(S_{j}\right)$, customers $\left(U_{u}\right)$ and a registration center $(R C)$. The generic architecture of remote-user authentication is shown in Fig. 1. The registration of each user and server with $R C$ take place on secure channel. Therefore, trust flows from RC to respective users and servers.

(c) The Author(s) 2020. This article is licensed under a Creative Commons Attribution 4.0 International License, which permits use, sharing, adaptation, distribution and reproduction in any medium or format, as long as you give appropriate credit to the original author(s) and the source, provide a link to the Creative Commons licence, and indicate if changes were made. The images or other third party material in this article are included in the article's Creative Commons licence, unless indicated otherwise in a credit line to the material. If material is not included in the article's Creative Commons licence and your intended use is not permitted by statutory regulation or exceeds the permitted use, you will need to obtain permission directly from the copyright holder. To view a copy of this licence, visit http://creativeco mmons.org/licenses/by/4.0/. 


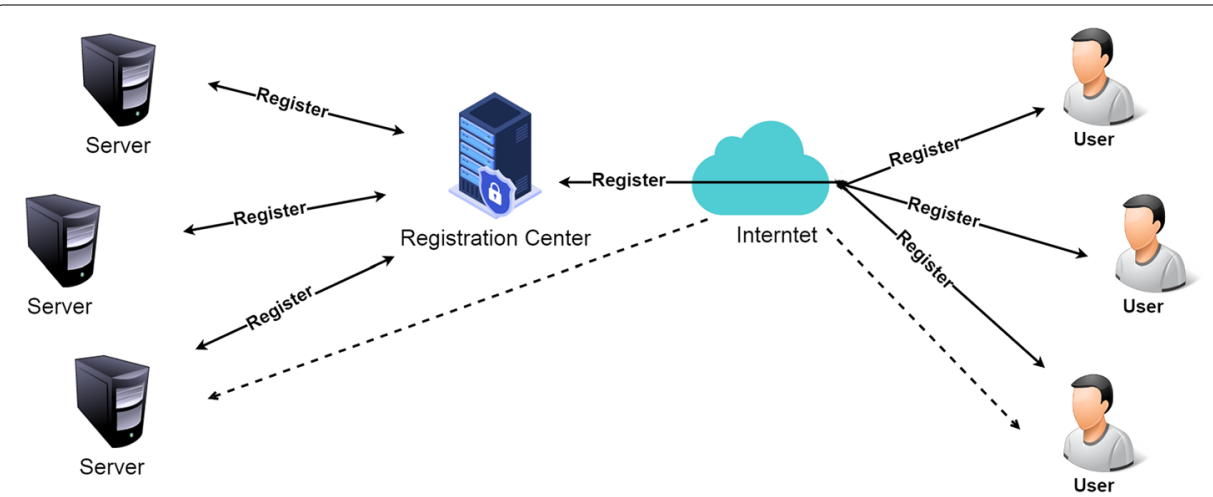

Fig. 1 Generic multi-server authentication environment

Thereafter, the customers are able to get advantages of the services provided by service providers.

Already, plenty of schemes have been introduced so far to achieve better efficiency and security [3-9]. However, it is realized on the basis of frequent security attacks that more stronger protocols need to be developed [10-14]. Initially, a key agreement protocol for MSA framework is presented early in 2000 by Lee and Chang [15]. Later, the protocol is found susceptible to impersonation and anonymity violation attacks [16]. After that, MSA scheme engaged by using RSA (Rivest - Shamir - Adleman)

Generic multi-server authentication environment

crypto-primitives and Lagrange interpolating polynomials, for a remote subscriber is presented by Tsaur [17]. The protocol [17] is compromised by password-guessing attack. Then, MSA protocol for a system of artificial neural network based on password is presented by Li et al. [18], which needs more time and higher cost. After that, ElGamal digital signature-dependent $M S A$ protocol is presented by Lin et al. [1]. However, it is realized for smart card dependent applications that the scheme is too expensive in terms of memory requirement. Thereafter, $M S A$ protocol based on symmetric crypto-system is presented by Juang [2], with an inherent scalability issue due to maintaining verifierrepository for each user at server end.

$M S A$ protocol is then introduced by Chang and Lee [19], which is found to be vulnerable for privileged-insider and server impersonation attacks [20,21]. Afterwards, another remote user verified key agreement protocol based on dynamic identity is presented by Liao and Wang [20] for MSA architecture. Hsiang and Shih [21] found that the protocol [20] is susceptible to spoofing and privileged insider attacks, and also introduced an enhanced protocol. Soon, Lee et al. [22] realized that the protocol [21] has no ability to attain agreement for mutual authentication and also introduced an modified scheme. However, Chen and Lee [23] observed that the protocol [24] is inadequate for smart card security and incompatible with two-factor authentication. Likewise, the protocol [24] also fails to prevent masquerading attack. Moreover, password change phase becomes more complex due to involvement of $R C$. Then, Irshad et al. [25] proves that the protocol [23] is insecure against smart card stolen attack that helps to reveal the session key and password. The protocol [23] is also vulnerable to trace and spoofing attacks. Later, due to the inefficiency and insecurity of the above mentioned schemes, many researchers have 
made improvements to the authentication method [26-28]. Additionally, some protocols have begun to use biometrics to ensure security [29]. The above discussion shows that designing the protocol for multi-server infrastructures to meet security requirements is a serious task. All current solutions are neither immune to all known attacks, nor they can guarantee the consumption of their own computations. Section III demonstrates our proposed scheme. Security and performance analysis are illustrated in Section IV and V, respectively. Presented work is concluded in last Section VI.

\section{Our contribution}

An anonymous three factor authentication protocol is introduced in this paper and the authentication of users with the help of biometric impression is enhanced. We encompass our contributions as follows.

1. First, we introduce an $E C C$ based three-factor user authenticated key-agreement protocol.

2. Second, if smart card can be forged by an adversary, then the environment of user cannot be secure. In our introduced protocol, the verification of biometric impression of users can be done by the client as well as by the server; in some specific applications it can provide security protection for specific requirements. RC and server have separate responsibilities, as $\mathrm{RC}$ is involved in authentication phase. $\mathrm{RC}$ retains the privacy of registration and server validates the client for further service providing; it can make the protocol more scalable for multi-server the architecture.

3. Finally, our protocol offers the mutual authentication for each pair of three participants (server, user and $R C$ ) for providing strong protection by identifying as possible replay messages.

\section{Preliminaries}

The hash functions, elliptic curve cryptography, adversarial model which is used in this paper are stated in this section. Whereas, Table 1 is presenting the common notations, used in rest of the article.

\section{Hash functions}

By taking an input string $O=H$ (String) of random size, a fixed size output is generated by hash. Generated output is called hash code. A little change in the value of string can cause a huge difference. Whereas, a secure one way hash function has following specifications:

- If the string is described, it is easy to find $O=H$ (String).

- It is impossible to find out the string, if $O=H$ (String) is illustrated.

- It is mundane task to distinguish input of String $1_{1}$ and String 2 so that $H\left(\right.$ String $\left._{1}\right)=H\left(\right.$ String $\left._{2}\right)$. This feature is called collision resistance.

Definition 1 (Characteristics of collision Resistance) Secure hash function $\mathrm{H}($.$) is$ predetermined for collision resistance. The possibility that an attacker $\mathcal{A}$ can find a 
Table 1 Common used notations

\begin{tabular}{ll}
\hline Common notations & blueElucidations \\
\hline $\mathcal{U}_{u}$ & $u_{\text {th }}$ user of the system \\
$\mathcal{R C}$ & Centralized registration center of the infrastructure \\
$I D_{u}$ & Specific user's identity \\
$P W_{u}$ & Specific user's password \\
$B_{u}$ & Biometric identity of specific user \\
$P I D_{u}$ & User's pseudo identity \\
$S C_{u}$ & Smart card issued to each specific user \\
$\mathcal{S}_{j}$ & jth service provider of the infrastructure \\
$I D_{j}$ & Identity of service provider \\
$x$ & Secret key of $\mathcal{R} \mathcal{C}$ \\
$p k_{R C}$ & Public key of $\mathcal{R C}$ \\
$S$ & Secret key of $\mathcal{S}_{j}$ \\
$p k_{S_{j}}$ & Public key of $\mathcal{S}_{j}$ \\
$E_{p}(e, f)$ & An elliptic curve \\
$P$ & Base point of the elliptic curve $E_{p}(e, f)$ \\
$H()$. & Function specified for Bio-hash \\
$h()$. & One-way digest function of hashing \\
$\|$ & Concatenation operator \\
$\oplus$ & XoR operator \\
\hline &
\end{tabular}

pair $\left(\right.$ String $_{1} \neq$ String $\left._{2}\right)$ as $H\left(\right.$ String $\left._{1}\right)=H\left(\right.$ String $\left._{2}\right)$ is separated as $\operatorname{Advs}_{\mathcal{A}}^{H A S H}(t)=$ $\operatorname{Prob}\left[\left(\right.\right.$ String $_{1}$, String $\left._{2}\right) \Leftarrow_{r} \mathcal{A}:\left(\right.$ String $_{1} \neq$ String $\left._{2}\right), H\left(\right.$ String $\left._{1}\right)=H\left(\right.$ String $\left.\left._{2}\right)\right], \quad$ where attacker is allowed to select a pair (String ${ }_{1}$, String $_{2}$ ) randomly. Attacker's perk is calculated against the randomly selections taken up with-in polynomial time $(t)$. The collision resistance conclude that $A d v s_{\mathcal{A}}^{H A S H}(t) \leq \epsilon$, whereas $\in>0$, is an enough tiny value.

\section{Elliptic-curve cryptography(ECC)}

The Elliptic-curve equation is defined in the form $E_{p}(e, f): c^{2}=d^{3}+e d+f$ over a prime finite field $(d, c) \in W_{P}^{*} \times W_{P}, e, f$ and $4 e^{3}+27 f^{2} \neq 0(\bmod \mathrm{P})$. Where $P$ is a selected huge prime number, the size of $P$ is $\geq 160$ bits. Scalar product is gained by repeated addition e.g. $n P=P+P+P+\ldots+P$ (ntimes), over a determined $t$ which a point on $E_{P}(e, f)$ and the multiplier $n$. The variables $(e, f, t, P, n)$ should be a part of limited field $F_{P}$. $E$ is supposed to be the abelian group. Whereas $O$, is stated as the ID's infinity point.

Definition 2 (Logarithmic issues in ECDLP) ECDLP: is given two specified points over $R, V \in E_{P}(e, f)$, calculate $n$ a scalar so that $R=n V$. The chances that attacker $\mathcal{A}$ can compute $n$ in polynomial time $(T)$ are described as $A d v s_{X}^{E C D L P}(T)=\operatorname{prob}\left[\left(X(R, V)=x: x x \in W_{P}\right)\right]$. ECDLP assumption concludes that $\operatorname{Advs}_{x}^{E C D L P}(T) \leq \epsilon$. 


\section{Adversarial model}

The familiar adversarial model is deliberated in this paper, as specified in [2, 30]. Where the following considerations are followed as per the expertise of the adversary Advs:

1. Advs have full control over the public communication channel. Advs is adept to eliminate, amend, rerun, interrupt or can send a new replicated message.

2. The information stored in the smart card can be excerpted by Advs, by doing power analysis.

3. Advs can be a deceitful or intruder user or service provider of the system.

4. The identities of registered servers and users are not private but familiar to insiders.

5. The attack on server cannot be launched by $A d v s$ because the server is assumed to be secured.

\section{Proposed scheme}

We propose an anonymous multi-server authentication protocol in this section. Although, proposed protocol brings more computation at server side, but server is usually assumed to have sufficient resources. Therefore, server can easily manages these extra computations in order to lower the computation cost on user side. The scheme is based on multi-server architecture which involves user $\left(U_{u}\right)$, server $\left(S_{j}\right)$ and registration center $(R C)$. RC provides facility for user registration and further helps to give services from server. $R C$ selects its master secret key $x$ to register all users. Like former schemes, the proposed scheme has also three stages: the authentication, registration and password change stage. The proposed protocol is shown in Fig. 2 and described in the below subsections.

\section{Server registration phase}

To become legitimate server $S_{j}$, the server needs to register with $R C$ by following these steps.

SR Step1: $S_{j}$ selects his identity $I D_{j}$ and sends to RC through secure channel.

SR Step2: After receiving $I D_{j}$, RC calculates $s=h\left(I D_{j} \| x\right), p k_{S_{j}}=s P$ and $p k_{R C}=x P$ where $x$ is secret key maintained by $R C$.

SR Step3: After that, $R C$ sends $s, p k_{S_{j}}, p k_{R C}$ to server $S_{j}$ and aborts the registration.

\section{User registration phase}

$U_{u}$ performs the following operations with $\mathrm{RC}$ to become the legal user of the network.

UR Step1: User selects his identity $I D_{u}$, password $P W_{u}$, biometric impression $B_{u}$ and generates an arbitrary nonce $a$. Then user determines $M=H\left(I D_{u} \| B_{u}\right)$, 
Server $\left(S_{j}\right)$

Registration Centre $(\mathcal{R C})$

Each server chooses $I D_{j}$

$\left\{I D_{j}\right\}$

$\left\{I D_{j}\right\}$

Computes $s=h\left(I D_{j} \| x\right)$

$p k_{S_{j}}=s P$

$p k_{R C}=x P$

$\left\{s, p k_{S_{j}}, p k_{R C}\right\}$

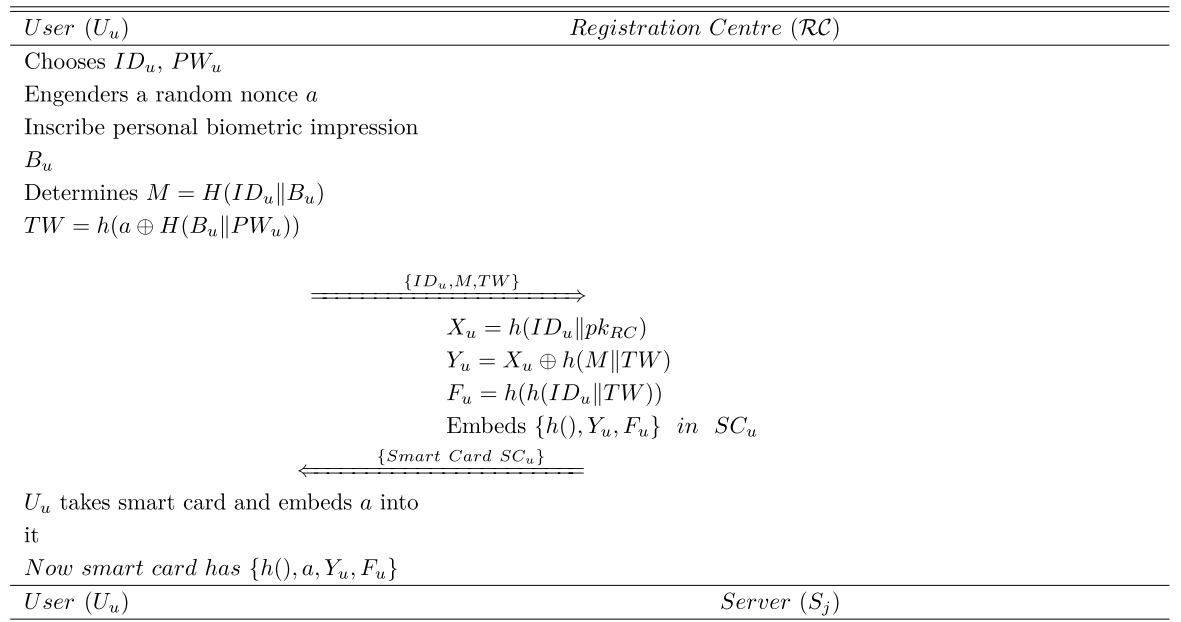

\section{Authentication Phase}

Input its smart-card in specific card-

reader

Enter $I D_{u}$ and $P W_{u}$ and biometric im-

pression $B_{u}$

Then $S C_{u}$ computes

$T W=h\left(a \oplus H\left(B_{u} \| P W_{u}\right)\right)$

Determine $F_{u} \stackrel{?}{=} h\left(h\left(I D_{u} \| T W\right)\right)$

$M=H\left(I D_{u} \| B_{u}\right)$

Generates random number $C_{u}$ and com-

putes

$O_{p}=C_{u} p k_{S_{j}}=C_{u} s P$

$P I D_{u}=C_{u} P \oplus I D_{u}$

$X_{u}^{\prime}=Y_{u} \oplus h(M \| T W)$

$D I D_{u}=h\left(I D_{u}\left\|X_{u}\right\| C_{u} P\right)$

$M_{1}=\left\{P I D_{u}, D I D_{u}, O_{p}\right\}$
$s^{-1} O_{p}=C_{u} P$

$I D_{u}=C_{u} P \oplus P I D_{u}$

$X_{u}=h\left(I D_{u} \| p k_{R C}\right)$

$D I D_{u} \stackrel{?}{=} h\left(I D_{u}\left\|X_{u}\right\| C_{u} P\right)$

Generates random number $D_{j}$

$T_{u}=h\left(I D_{u} \| X_{u}\right)$

$V_{j}=D_{j} \oplus X_{u}$

$Q_{u j}=h\left(I D_{u}\left\|T_{u}\right\| C_{u} P\left\|D_{j}\right\| X_{u} \| I D_{j}\right)$

$M_{2}=\left\{Q_{u j}, V_{j}\right\}$

$D_{j}=V_{j} \oplus X_{u}$

$h\left(I D_{u}\left\|h\left(I D_{u} \| X_{u}^{\prime}\right)\right\| C_{u} P\left\|D_{j}\right\| X_{u}^{\prime} \| I D_{j}\right) \stackrel{?}{=}$

$Q_{u j}$

$S K_{u j}=h\left(I D_{u}\left\|C_{u} P\right\| D_{j}\left\|X_{u}^{\prime}\right\| I D_{j}\right)$

$Z_{u j}=h\left(S K_{u j}\left\|I D_{u}\right\| D_{j}\left\|X_{u}^{\prime}\right\| I D_{j}\right)$

$M_{3}=\left\{Z_{u j}\right\}$

Checks $\quad S K_{u j}$

$h\left(I D_{u}\left\|C_{u} P\right\| D_{j}\left\|X_{u}\right\| I D_{j}\right)$

$h\left(S K_{u j}\left\|I D_{u}\right\| C_{u} P\left\|X_{u}\right\| I D_{j}\right) \stackrel{?}{=} Z_{u j}$

Common Exchanged Key $=S K_{u j}=h\left(I D_{u}\left\|C_{u} P\right\| D_{j}\left\|X_{u}\right\| I D_{j}\right)$

Fig. 2 Proposed Scheme 
$T W=h\left(a \oplus H\left(B_{u} \| P W_{u}\right)\right)$ and sends $I D_{u}, M, T W$ to $\mathrm{RC}$ for completing the registration.

UR Step2: After that RC determines $X_{u}=h\left(I D_{u} \| p k_{R C}\right), Y_{u}=X_{u} \oplus h(M \| T W)$ and $F_{u}=h\left(h\left(I D_{u} \| T W\right)\right)$, then $\mathrm{RC}$ stores $h(), Y_{u}, F_{u}$ in smart card and sends $\left(S C_{u}\right)$ towards $U_{u}$.

UR Step3: $U_{u}$ further adds a number $a$ into $S C_{u}$. Now smart card have $\left\{h(), Y_{u}, F_{u}, a\right\}$.

\section{Login and authentication phase}

In this phase, authenticated access is granted to user $U_{u}$ for accessing service providers $S_{j} . U_{u}$ and $S_{j}$ authenticate themselves in following steps.

LAP Step1: $U_{u}$ inputs $I D_{u}$, password $P W_{u}$ and scan biometric impression in scanner. Then smart card determines $T W=h\left(a \oplus H\left(B_{u} \| P W_{u}\right)\right)$ and checks whether $F_{u} \stackrel{?}{=} h\left(h\left(I D_{u} \| T W\right)\right)$. If yes, then determines $M=H\left(I D_{u} \| B_{u}\right), U_{u}$ creates a random number $C_{u}$ and computes $O_{p}=C_{u} p k_{S_{j}}=C_{u} s P, \quad P I D_{u}=C_{u} P \oplus I D_{u}$, $X_{u}^{\prime}=Y_{u} \oplus h(M \| T W) \quad$ and $\quad D I D_{u}=h\left(I D_{u}\left\|X_{u}^{\prime}\right\| C_{u} P\right)$. Then $\quad U_{u}$ sends $M_{1}=P I D_{u}, D I D_{u}, O_{p}$ to $S_{j}$.

LAP Step2: After receiving $M_{1}=P I D_{u}, D I D_{u}, O_{p}, S_{j}$ using his secret key $s$ computes $s^{-1} O_{p}=C_{u} P, I D_{u}=C_{u} P \oplus P I D_{u}$ and $X_{u}=h\left(I D_{u} \| p k_{R C}\right)$. After that $S_{j}$ checks $D I D_{u} \stackrel{?}{=} h\left(I D_{u}\left\|X_{u}\right\| C_{u} P\right)$. If it holds true, then $R C$ creates arbitrary nonce $D_{j}$ and determines $T_{u}=h\left(I D_{u} \| X_{u}\right), V_{j}=D_{j} \oplus X_{u}$ and $Q_{u j}=h\left(I D_{u}\left\|T_{u}\right\| C_{u} P\left\|D_{j}\right\| X_{u} \| I D_{j}\right)$. Subsequently, $S_{j}$ sends a message $M_{2}=Q_{u j}, V_{j}$ to $U_{u}$.

LAP Step3: $U_{u}$ determines $D_{j}=V_{j} \oplus X_{u}$ after receiving $M_{2}$ and checks $h\left(I D_{u}\left\|h\left(I D_{u} \| X_{u}^{\prime}\right)\right\| C_{u} P\left\|D_{j}\right\| X_{u}^{\prime} \| I D_{j}\right) \stackrel{?}{=} Q_{u j}$.

LAP Step4: If the $h\left(I D_{u}\left\|h\left(I D_{u} \| X_{u}^{\prime}\right)\right\| C_{u} P\left\|D_{j}\right\| X_{u}^{\prime} \| I D_{j}\right) \stackrel{?}{=} Q_{u j}$ holds true, $U_{u} \quad$ further determines $S K_{u j}=h\left(I D_{u}\left\|C_{u} P\right\| D_{j}\left\|X_{u}^{\prime}\right\| I D_{j}\right)$ and computes $Z_{u j}=h\left(S K_{u j}\left\|I D_{u}\right\| D_{j}\left\|X_{u}^{\prime}\right\| I D_{j}\right) . U_{u}$ sends $M_{3}=Z_{u j}$ towards $S_{j}$ so that it can check the challenge based on $D_{j}$

LAP Step5: After getting $M_{3}$, the server $S_{j}$ determines $S K_{u j}=h\left(I D_{u}\left\|C_{u} P\right\| D_{j}\left\|X_{u}\right\| I D_{j}\right)$. After that, it justifies the equation i.e. $h\left(S K_{u j}\left\|I D_{u}\right\| C_{u} P\left\|X_{u}\right\| I D_{j}\right) \stackrel{?}{=} Z_{u j}$. Finally, on successful justification, server exchanges the session-key $S K$ with user as $h\left(I D_{u}\left\|C_{u} P\right\| D_{j}\left\|X_{u}\right\| I D_{j}\right)$. The description of this protocol can be endorsed from Fig. 2.

\section{Password changing phase}

$U_{u}$ may change his password into another new password $\left(P W_{u}^{n}\right)$ by using these steps. These steps are as follows:

PC Step1: Initially, user input identity $I D_{u}^{*}$, password $\left(P W_{u}^{*}\right)$ and scan biometric impression factor after inserting smart card $(S C)$ into reader. After that, $S C$ determines $T W=h\left(a \oplus h\left(B_{u} \| P W_{u}\right)\right)$ and justify $F_{u} \stackrel{?}{=} h\left(h\left(I D_{u} \| T W\right)\right)$. If it holds true then user will follow next step. 
PC Step2: Subsequently, $S C$ determines $T W=h\left(a \oplus h\left(B_{u} \| P W_{u}\right)\right)$ and calculates $X_{u}=h\left(I D_{u} \| T W\right), Y_{u} *=X_{u} \oplus h(M \| T W)$.

PC Step3: Afterwards, when user will change password $\left(P W i^{n}\right)$. The smart card then determines $T W=h\left(a \oplus h\left(B_{u} \| P W_{u}^{n}\right)\right), \quad Y_{u}^{n}=X_{u} \oplus h\left(M \| T W^{\prime}\right), \quad$ and $F_{u}^{n}=h\left(h\left(I D_{u} \| T W\right)\right)$.

PC Step4: Then the values $X_{u}, Y_{u}$, and $F_{u}$ are changed by $X_{u}^{n}, Y_{u}^{n}, F_{u}^{n}$ in smart card.

\section{Revocation/re-registration phase}

In this section, we show that if $U_{u}$ 's smart card has been stolen or his account has been revoked then he can request for reregistration. For this purpose he must follow subsequent steps:

RP Step1: $\left(U_{u}\right)$ engenders a random number $a^{*}$, a new password $P W_{u}^{*}$, and biometric $B_{u}^{*}$ of his/her own choice. Then calculates $M^{*}=H\left(I D_{u} \| B_{u}^{*}\right)$ and $T W^{*}=h\left(a^{*} \oplus H\left(B_{u}^{*} \| P W_{u}^{*}\right)\right)$ and submits request message $\left\{I D_{u}, M^{*}, T W^{*}\right\}$ to the registration centre $(\mathcal{R C})$ through a secure path.

RP Step2: On receiving request message $\left\{I D_{u}, M^{*}, T W^{*}\right\}$ from $\left(U_{u}\right), \mathcal{R C}$ will first verify whether $\left(U_{u}\right)$ is already a registered user or not from the verifier table. If a match is not found in the database, the $\mathcal{R C}$ will reject the request.

RP Step3: $\mathcal{R C}$ then embeds the security parameters $\left\{h(), Y_{u}^{*}, F_{u}^{*}\right\}$ in a new $S C_{u}^{*}$ into the smart card and sends the new smart card to the user $\left(U_{u}\right)$ through secure path.

RP Step4: $U_{u}$ takes new smart card $S C_{u}^{*}$ and embeds $a^{*}$ into it. The phase is shown in Fig. 3.

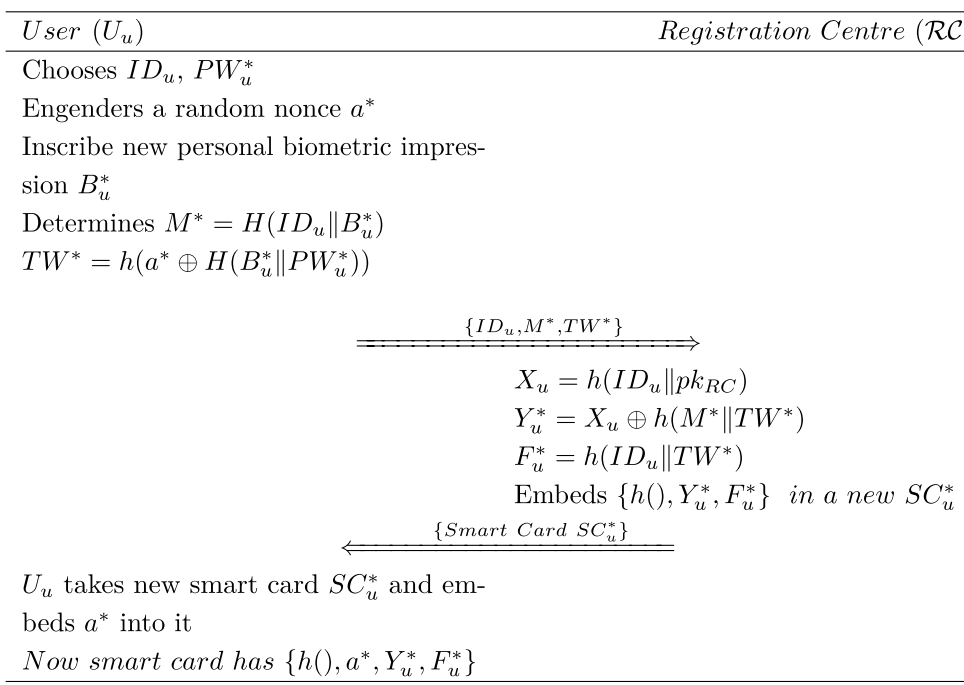

Fig. 3 Revocation phase 


\section{Security analysis}

In this section, informal and formal security analysis are presented. The security analysis highlights that the proposed scheme is safe and secure against various possible attacks.

\section{Informal security}

In this section, a comprehensive informal security analysis of contributed protocol is presented.

\section{Correct notion of user anonymity}

In several authentication schemes for multi-server environment, the server is usually unable to identify the identity of a user requesting for login. In our view, such notion of perfect anonymity is erroneous and not desirable in any environment, because if the server is unable to know a user's identity, he will be unable to provide the specific services to the user. In fact in this, any user can continue to get the services provided by the service provider even if he is not registered to the network or his lease has been expired. However, in proposed protocol, instead of user's identity $I D_{u}$, a dynamic-pseudo identity $P I D_{u}$ is sent during each authentication request message, to $S_{j}$. Furthermore, user's identity $I D_{u}$ can only be extracted using server's private key $s$. In addition, by analyzing two different session, an adversary will remain unable to guess whether the same user has initiated session. Hence, in this way our introduced protocol provides user's anonymity and untraceability.

\section{Replay attack}

In this flaw, the retrieved messages are restored without endure transformation to deceive any legitimate user [31-34]. Adversary can get the parameters $P I D_{u}, D I D_{u}, O_{p}, Q_{u j}, T_{u}, V_{j}$ and try to endure these parameters in request to forge the legal member. However, if an adversary retrieves contents, he cannot initiate an attack because $C_{u}$ and $D_{j}$ is created by legitimate member for every session. Similarly, if an adversary endeavors to replay $M_{1}=P I D_{u}, D I D_{u}, O_{p}$ toward server, server verifies the validity of user in $M_{3}$, in reply to the challenge based on $D_{j}$. Synchronously, the legitimate user validates $S_{j}$ in $M_{2}$ to response to the $M_{1}$ based challenge $C_{u}$. Hence the contributed protocol thwart replay attack.

\section{Stolen smart card attack with offline dictionary}

In stolen smart card attack with offline dictionary, the attacker tries different sequences of dictionary ingredients using stolen $S C$ credentials [35-37]. An attacker may attempts to exploit with its feasible parameters of $S C$ i.e $h(), Y_{u}, F_{u}$. For estimating the $P W_{u}$ from $Y_{u}$ and $F_{u}$ parameters, adversary needs to perceive $I D_{u}, a$ and $B_{u}$ to estimate $P W_{u}$ from $T W$ where $T W=h\left(a \oplus h\left(B_{u} \| P W_{u}\right)\right)$. Furthermore, this attack cannot initiate in polynomial time using smart card. 


\section{Known-key security}

Known-key security provides the confidentiality of private keys even with exposed session key for a particular session [38, 39]. Given that the specific session-key $S K_{u j}=h\left(I D_{u}\left\|C_{u} P\right\| D_{j}\left\|X_{u}\right\| I D_{j}\right)$ does not hold $U_{u}^{\prime} s$ password $P W_{u}^{\prime} s$ as a parameter. Owing it to, the adversary may not discover the parameters from derived session key. Hence, the contributed protocol offers known-key security.

\section{Mutual authentication}

Mutual authentication is provided by the enhanced scheme because the legitimate participants verify each other and thus it ensure mutual authentication strongly. This property makes our protocol secure and provides the early detection of possible attacks like replay attacks.

\section{Masquerading attack}

According to this attack, an attacker can masquerade one member of a specific session, if it reveals another member's key of the current session. The contributed protocol is immune to key-compromise impersonation threat in contrary to scheme, [23] as the contents of stolen card will not help the attacker to get other constructive parameters, such as $X_{u}$. Hence, the attacker cannot obtain newly generated $Q_{u j}$ factor and ultimately impersonation attack cannot be initiated.

\section{Stolen verifier attack}

The adversary misuses valued data which is stored at server's side and user's privates like passwords or other parameter, masquerade as legal users. The contributed protocol offers mutual authentication without maintaining repository on $S_{j}$ and RC's side. This shows that our scheme is withstand stolen verifier attack.

\section{Password guessing attack}

The guessing attack is applicable, if an adversary accesses the parameters $P I D_{u}, D I D_{u}, O_{p}, Q_{u j}, T_{u}, V_{j}$ on little analysis of any open channel. Nonetheless, an adversary cannot extract the password, after all it is not use as a factor for the computation of any contents, hence it minimizes the chances of estimating the consistent factors.

\section{Modification attacks}

The adversary changes the retrieving parameters and submit to promise party. In case, the scheme is designed to resist against modification threat. If the adversary attempts to change the public contents $P I D_{u}, D I D_{u}, O_{p}, Q_{u j}, T_{u}, V_{j}$, adversary will not able reassemble following parameters $P I D_{u}, D I D_{u}, O_{p}$ by introducing recent session arbitrary variables, since to assemble these parameters acquires the information of secret key and $X_{u}$ which knows to legal member. Consequently, the legal member can expose any venomous member easily. So, the enhanced scheme can easily discourage this attack. 


\section{Formal security analysis}

We have described model of security for presented protocol in this section. Furthermore, using given model of security the presented protocol is proved safe against known attacks. At the end, the proposed protocol is described to fulfill all the necessary requirements that relates to the security of the presented protocol.

Theorem THM1 Consider $D_{i}$ as a uniformly distributed dictionary consists of various possible passwords. $|D|$ denotes the size of $D_{i}$. Consider $A$ as an adversary against semantic security within a time bound $t$. Suppose a ECCDH problem stands, then we have

$D_{i}$ is considered as evenly distributed dictionary which consists of numerous passwords that can be possible. The size of $D_{i}$ is denoted by $|D| . A$ is considered as an adversary against syntactic security in a time bound $\mathrm{t}$. If a $E C C D H$ problem occurs, then we have

$$
\begin{aligned}
A_{\Pi, D}(A) \leq & \frac{\left(q_{h s h}+q_{\text {exe }}\right)^{2}}{2 p}+\frac{q_{h s h}^{2}}{p} \\
& +\frac{q_{h s h}}{p}+q_{h s h} A_{\Pi}^{E C C D H}(A) \\
& +\frac{q_{h s h}}{p}+\frac{q_{s h d}^{2}}{D}
\end{aligned}
$$

where the possibility of solving the $E C C D H$ problem by $A$, is denoted by $A_{\Pi}^{E C C D H}$. The number of Execute, Random-oracle and Send query are $\left\{q_{\text {exe }}, q_{h s h}, q_{\text {snd }}\right\}$, respectively.

Proof In order to give the proof of Theorem THM1, six composite games are considered from game $G_{1}$ to $G_{6}$. The game will be started where the real attack is simulated and a game will be ended where adversary $A$ has no advantage. The possibility of successfully guessing the random bit $b$ in test-query by $A$ is denoted by $S u c_{i}$ for each game $G_{i}$, where $1 \leq i \leq 6$.

GAME $G_{1}$ : In this random oracle model, the real attacks are simulated with the help of this game. In game $G_{1}$, every instance like $U_{u}, S_{j}$ and $R C$ will be modeled as authentic executions. As per the definition of $S u c 1$, we get following equation.

$$
A_{\Pi, D}^{E C C D H}(A)=2 \operatorname{Pr}(\operatorname{Suc} 1)-1 \text {. }
$$

GAME $G_{2}$ : Multiple oracles like hash oracle $h$ Execute, Corrupt, Reveal, Send and Test are simulated with $G_{2}$. Hash oracle is simulated by game $G_{2}$ by maintaining a hash list $h_{\text {list }}, h_{\text {list }}$ comprises on queries entries as (input, output). When a hash query is answered by hash oracle, then it returns the corresponding output if there is any existing query (input, output) in $h_{\text {list }}$, else it will return value from 0, 1. Moreover, corrupt, reveal, send and Test queries will be run as real attacks. Thread model is used to specify the actual actions of all these queries. This simulation indicates that game $G_{2}$ is perfectly secured from the real attacks. Thus, we have

$\operatorname{Pr}(\operatorname{Suc} 2)=\operatorname{Pr}(\operatorname{Suc} 1)$ 
GAME $G_{3}$ : This game consists on all possible executions of ROM as elaborated in game $G_{2}$ except that it will be discarded when some collision occured in the simulation of all hash queries, that are inquired by the adversary $A$. So, this game helps to avoid from collision to be occurred in ciphertext, password and output of Send-queries. By the definition of birthday paradox, the chances of occuring collision in hash oracle is $\frac{q_{e x e}^{2}}{2 p}$. Thats why the chances of occuring collision in game $G_{3}$ is $\frac{\left(q_{h s h}+q_{\text {exe }}\right)^{2}}{2 p}$. For this simulations, we achieved following equation

$$
|\operatorname{Pr}(\operatorname{Suc} 3)-\operatorname{Pr}(\operatorname{Suc} 2)| \leq \frac{\left(q_{h s h}+q_{\text {exe }}\right)^{2}}{2 p}+\frac{q_{\text {exe }}}{2 p} .
$$

GAME $G_{4}$ : This game consists on all possible executions of ROM as elaborated in game $G_{3}$ but it will be discarded after the successful guessing of $X_{u}$ by adversary $A$ without asking the hash oracle $h$. This game is similar all previous games unless the instances $\Pi_{U}^{i}$ and $\Pi_{S}^{j} S_{j}$ reject the actual authentication value. From game $G_{4}$, we get following equation

$$
|\operatorname{Pr}(\operatorname{Suc} 4)-\operatorname{Pr}(\operatorname{Suc} 3)| \leq \frac{q h s h}{p}
$$

GAME $G_{5}$ : This game indicates that if adversary guesses the session key directly without knowing and inquiring about hash oracle $h$ then this game will be terminated. It enables the session key to be independent with $\left\{P W_{u}, B_{u}\right\}$ and random numbers as well as point multiplication $C_{u}, D_{j}, P$. $G_{4}$. This game will be aborted after the inquiring common value $X_{u}$. Thus, $A_{\Pi}^{E C C D H}(A) \leq \frac{1}{q_{h s h}}|\operatorname{Pr}(\operatorname{Suc5})-\operatorname{Pr}(\operatorname{Suc} 4)|-\frac{1}{p}$ and we have

$$
|\operatorname{Pr}(\operatorname{Suc5})-\operatorname{Pr}(\operatorname{Suc} 4)| \leq q_{h} A_{\Pi}^{E C C D H}(A)+\frac{q_{h s h}}{p} .
$$

GAME $G_{6}$ : This game consists on all possible executions of ROM as elaborated in game $G_{5}$ except the rule if follow in Test query. $G_{5}$ will be aborted when $A$ queries about hash oracle that is identical values $C_{u}, D_{j}, P$. The chance of adversary $A$ getting the correct session-key by hash-query is at most $\frac{q_{h s h}^{2}}{2 p}$. Thus, we have

$$
|\operatorname{Pr}(\operatorname{Suc} 6)-\operatorname{Pr}(\operatorname{Suc} 5)| \leq \frac{q_{h s h}^{2}}{2 p} .
$$

Until adversary $A$ does not enter correct value into the random oracle $h$, the random oracle will remain indistinguishable against real attack. That's why $A$ does not have any advantage of identifying the legal session key from random oracle attempt. Furthermore, when corrupt query is performed, not more than 3 queries can be performed simultaneously. It means that if smart card corrupt and biometric corrupt $\left(\Pi_{U}^{i}, 3\right),\left(\Pi_{U}^{i}, 4\right)$ are performed then password corrupt $\left(\left(\Pi_{U}^{i}, 2\right)\right)$ cannot be performed this is the reason that success rate of off-line password guessing attack is $\frac{q_{\text {snd }}^{2}}{D}$. By combining all the equations from $G_{1}$ to $G_{6}$, we get following equation 


$$
\begin{aligned}
A_{\Pi, D}(A) \leq & \frac{\left(q_{h s h}+q_{\text {exe }}\right)^{2}}{2 p}+\frac{q_{h s h}^{2}}{p} \\
& +\frac{q_{h s h}}{p}+q_{h} A_{\Pi}^{E C C D H}(A) \\
& +\frac{q_{h s h}}{p}+\frac{q_{s n d}^{2}}{D}
\end{aligned}
$$

\section{Performance analysis}

In this section, the robustness of proposed protocol is assessed with respect to other schemes [20-23, 25] based on multi server architecture. The security traits and the scrutiny of defending to numerous attacks for different schemes are described in Table 2, in which the proposed protocol is signified as a strong corroborated key-agreement in contrast to former schemes. Table 2 presents the analysis of our schemes with related schemes [20-23, 25]. As per the analysis, we can conclude that our protocol is more secure than $[20-23,25]$. All these protocols depend upon hash-based symmetric cryp-

\begin{tabular}{|c|c|c|c|c|c|c|}
\hline Scheme: & Proposed & $\begin{array}{l}\text { Liao } \\
\text { and Wang } \\
{[20]}\end{array}$ & $\begin{array}{l}\text { Hsiang } \\
\text { and Shih } \\
{[21]}\end{array}$ & Lee et al. [22] & $\begin{array}{l}\text { Chen } \\
\text { and Lee } \\
{[23]}\end{array}$ & $\begin{array}{l}\text { Irshad } \\
\text { et al. } \\
{[25]}\end{array}$ \\
\hline Immune to smart card stolen attack & Yes & Yes & Yes & Yes & No & Yes \\
\hline Efficient password modification & Yes & Yes & Yes & No & No & Yes \\
\hline Ensuring anonymity & Yes & Yes & Yes & Yes & Yes & No \\
\hline Immune to insider attack & Yes & No & Yes & Yes & Yes & Yes \\
\hline Immune to trace attack & Yes & Yes & Yes & Yes & No & Yes \\
\hline Immune to impersonation attack & Yes & No & No & No & No & Yes \\
\hline Support mutual authentication & Yes & No & No & No & Yes & Yes \\
\hline Repair ability & Yes & Yes & No & No & Yes & Yes \\
\hline Supports session key security & Yes & Yes & Yes & Yes & No & Yes \\
\hline $\begin{array}{l}\text { Immune to offline password guess- } \\
\text { ing attack }\end{array}$ & Yes & Yes & Yes & No & Yes & Yes \\
\hline Immune to $\mathrm{KCl}$ attack & Yes & Yes & No & Yes & No & Yes \\
\hline
\end{tabular}
tography and similar in nature.

Table 2 Comparison of security parameters

\begin{tabular}{|c|c|c|c|c|}
\hline Protocols & Computational cost & $\operatorname{Energy}(m J)$ & $\begin{array}{l}\text { Communication } \\
\text { cost(bits) }\end{array}$ & $\begin{array}{l}\text { Storage } \\
\text { cost(bits) }\end{array}$ \\
\hline Proposed & $8 T_{h}+7 T_{h}+1 T_{H}=0.0120 \mathrm{~ms}$ & 0.91 & 1920 & 1440 \\
\hline Liao and Wang [20] & $9 T_{h}+9 T_{h}=0.0117 \mathrm{~ms}$ & 0.98 & 2944 & 1024 \\
\hline Hsiang and Shih [21] & $9 T_{h}+12 T_{h}=0.0132 \mathrm{~ms}$ & 1.10 & 3296 & 1440 \\
\hline Lee et al. [22] & $9 T_{h}+10 T_{h}=0.0125 \mathrm{~ms}$ & 1.05 & 3296 & 1440 \\
\hline Chen and Lee [23] & $12 T_{h}+10 T_{h}=.0146 \mathrm{~ms}$ & 1.24 & 3252 & 1440 \\
\hline Irshad et al. [25] & $13 T_{h}+13 T_{h}+3 T_{H}=0.0169 \mathrm{~ms}$ & 1.64 & 3872 & 1952 \\
\hline
\end{tabular}

Table 3 Comparison of energy and computational, communication and storage costs 

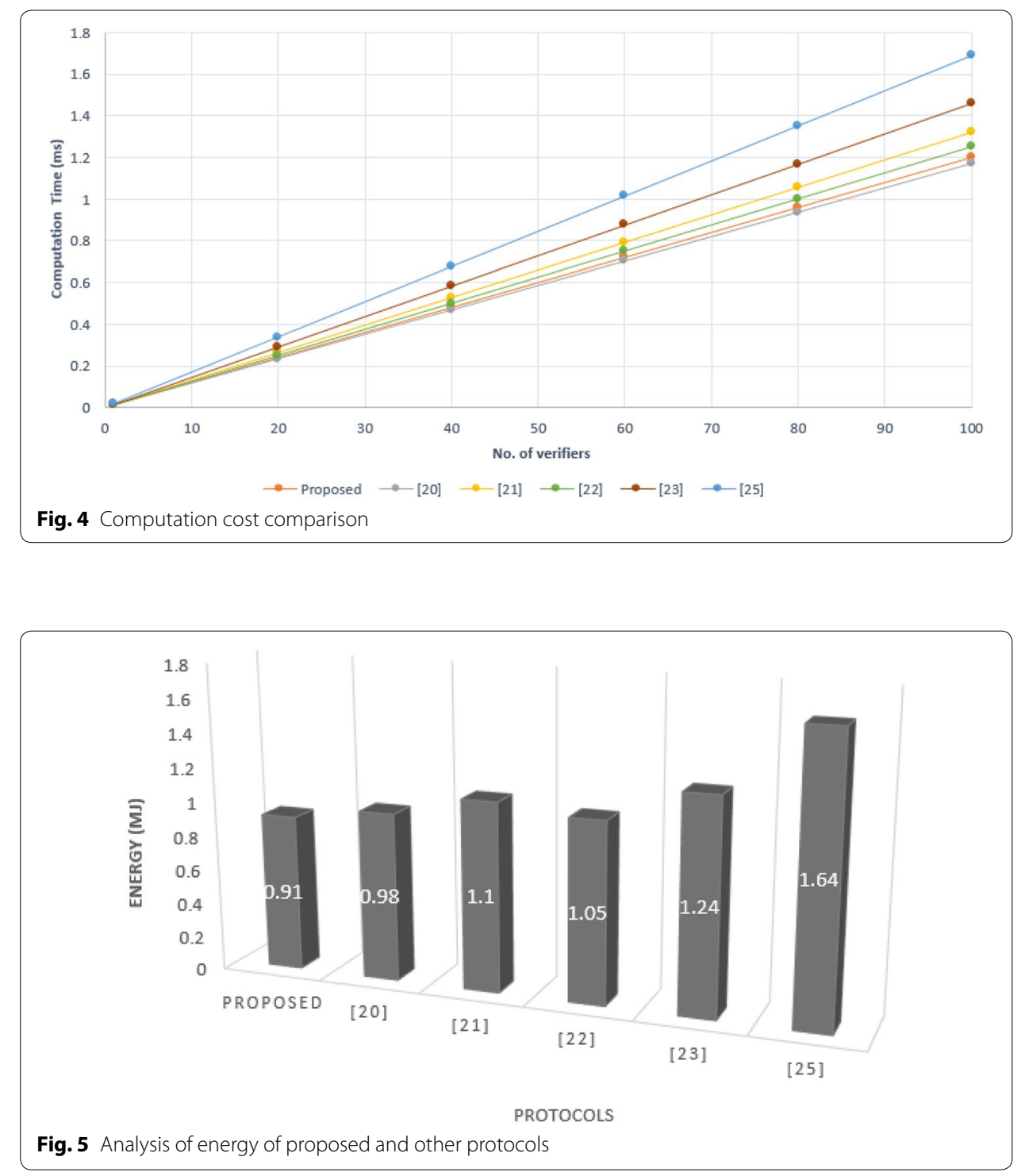

Later, the performance analysis of our authenticated protocol in terms of cost has been analyzed. The specification and description for the implementation is as follows; the implementation of the cryptographic functions $\left(T_{\oplus}, T_{\|}, T_{h(.)}, P_{m}\right)$ is done by using py-crypto library inside ubuntu 19.04, with $16.0 \mathrm{~GB}$ RAM and $3.60 \mathrm{GHz}$ processor core $i 7$ with the help of python programming language. The execution of authentication scheme is done under same assumptions for 10 times by averaging. Some functions like $\left(T_{\|}, T_{\oplus}\right)$ have not been considered because they acquires negligible execution time. The execution time for $h(),. H($.$) and point multiplication operations$ is $0.0120 \mathrm{~ms}, 0.015 \mathrm{~ms}$ and $0.02957 \mathrm{~ms}$, respectively. The communication, energy requirements, storage and computation cost of our scheme with respect to related protocols is presented in Table 3. The time for execution of considered cryptographic functions are assumed as follows: 


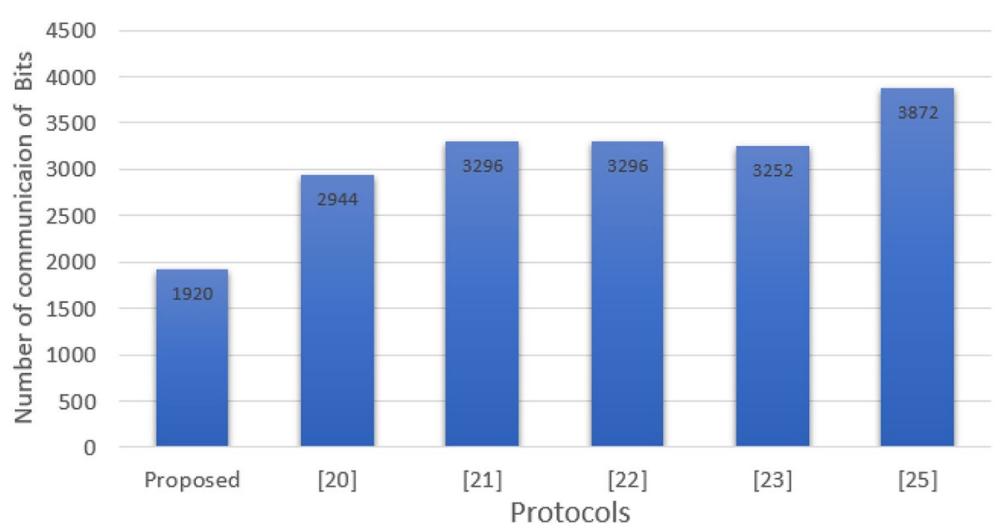

Fig. 6 Analysis of communication cost between proposed and related protocols

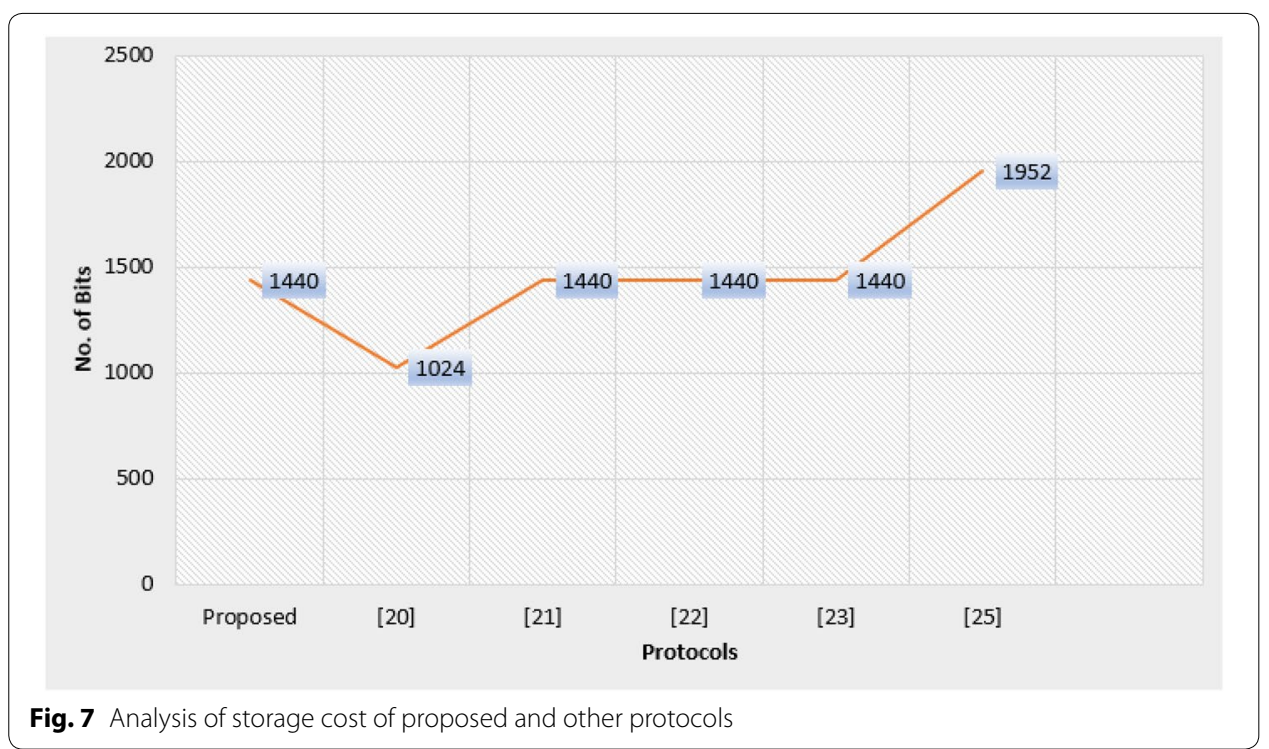

- Execution time for one way hash function is $E_{h}=0.0120 \mathrm{~ms}$.

- Execution time for one way bio-hash function is $E_{H}=0.015 \mathrm{~ms}$.

- Execution time for point multiplication $E_{p m}=0.02957 \mathrm{~ms}$.

It is observed the computation cost of our proposed scheme is higher than [20-23, 25] schemes but it offers aided security features. Furthermore, the mandatory security objectives are achieved by our protocol in less cost than Hsiang and Shih's scheme. Moreover, the proposed protocol (contrasting with former protocols) is secure to smart card stolen, password guessing and insider attacks.

We have determined cost comparison in Table 3, which are later elaborated by drawing Figs. 4, 5, 6 and 7. The cost of computation for proposed and relevant schemes is showcased in Fig. 4. The number of verifiers of our proposed and existing protocols are shown horizontally and required computation time according to the number of verifiers 
is shown vertically in the graph. It can be observed that computation cost of our protocol is far less than the related schemes..

Energy consumption can be calculated as $E_{c}=T_{c c} P_{C P U}$, where $T_{c c}$ is the total computation cost for a single hash function $(0.054 \mathrm{~mJ}), P_{C P U}$ is the maximum power $(65 \mathrm{~W})$ of CPU and $E_{c}$ is the energy consumption [40]. Power consumption can be used to give a rough estimate of energy consumed during computation. Moreover, we have examined the protocol with respect to energy consumption by considering computation cost of energy for SHA-1 as $0.54 \mathrm{~mJ}$ for single byte [41] shown in Fig. 5 . By Considering this, the consumption of energy for the [20-23, 25] and our scheme amounts to $1.64 \mathrm{~mJ}, 0.98 \mathrm{~mJ}$, $1.10 \mathrm{~mJ}, 1.05 \mathrm{~mJ}, 1.24 \mathrm{~mJ}$ and $0.91 \mathrm{~mJ}$, respectively. The final energy consumption determined values of proposed and related schemes are given in Table 3. Hence, it can be calculated that the energy consumption of proposed scheme is less than related schemes.

The assumptions made for determining the communication and storage cost are as follows: 160 bits are reserved for random nonce, timestamps, password and identity, 256 bits are for one way hash function and for public key, 512 bits. The calculations of storage and communication cost of our and related schemes on the basis of above mentioned assumptions are presented in Table 3.

The cost of communication for proposed and relevant schemes is presented in Fig. 6 . The proposed and related schemes are given horizontally, while the required number of communication bits are shown vertically in the graph. It is observed that the number of communication bits of proposed scheme is slightly greater than related schemes but our scheme provides more security traits. The storage cost of proposed and related schemes is displayed in Fig. 7. The vertically labeled values on the graph are for the required number of storage bits, while proposed and related schemes are listed horizontally.

The storage bits of our scheme is slightly greater than the related protocol. This is just because of providing more security features for making secure protocol. After analyzing Tables 2 and 3, we can say that the computation time of our scheme is less than the related schemes and also provides more security traits with slightly higher communication and storage costs.

\section{Conclusion}

The robustness of multi-server authentication is observed as an important requisite for the current remote based authentication paradigm. Recently, extensive research has been conducted for developing robust authentication protocols for multi-server authentication environment. In this paper, we proposed an anonymous multi-server authentication scheme. The flaws of previous schemes are kept in mind in order to develop the proposed scheme with enhanced security features. The analysis of performance evaluation and formal security is also described in this paper against various schemes. This analysis also shows that our scheme provides more security features.

Acknowledgements

Not applicable

Authors' contributions

MAA, ZG and KM analyzed the requirement of the security for Multi-server Infrastructure, designed the framework, conducted the experiment and drafted the manuscript. Also, KM gave full support in conducting the experiment and assisted in draft work and revised the manuscript. SK contributed by reviewing the work done and in revising the content of the manuscript. In addition, all the work is done in SK's supervision. KA has helped us in major revision according to the comments of reviewers. CMC coordinated the whole study. All authors read and approved the final manuscript. 
Availability of data and materials

It is theoretical work no data is needed.

\section{Competing interests}

The authors proclaim, they have no competing interests.

\section{Author details}

${ }^{1}$ Department of Computer Science, COMSATS University Islamabad, Sahiwal Campus, Sahiwal 57000, Pakistan.

2 Department of Mathematics, Chaudhary Charan Singh University, Meerut, Uttar Pradesh 250004, India. ${ }^{3}$ Department of Computer Science and Engineering, Bhagwati Institute of Technology and Science, Ghaziabad 201302, India. ${ }^{4}$ College of Computer Science and Engineering, Shandong University of Science and Technology, Qingdao 266590, China.

Received: 3 January 2020 Accepted: 17 April 2020

Published online: 15 May 2020

\section{References}

1. Lin I-C, Hwang M-S, Li L-H (2003) A new remote user authentication scheme for multi-server architecture. Fut Gen Comput Syst 19(1):13-22

2. Juang W-S (2004) Efficient password authenticated key agreement using smart cards. Comput Secur 23(2):167-173

3. Chen C-M, Xiang B, Liu Y, Wang K-H (2019) A secure authentication protocol for internet of vehicles. IEEE Access 7:12047-12057

4. Kumari S, Chaudhary P, Chen C-M, Khan MK (2019) Questioning key compromise attack on ostad sharif et al authentication and session key generation scheme for healthcare applications. IEEE Access 7:39717-39720

5. Kumari S, Li X, Wu F, Das AK, Choo K-KR, Shen J (2017) Design of a provably secure biometrics-based multi-cloudserver authentication scheme. Fut Gen Comput Syst 68:320-330

6. Kumari S, Khan MK, Atiquzzaman M (2015) User authentication schemes for wireless sensor networks: A review. Ad Hoc Netw 27:159-194

7. Kumari S, Li X, Wu F, Das AK, Arshad H, Khan MK (2016) A user friendly mutual authentication and key agreement scheme for wireless sensor networks using chaotic maps. Fut Gen Comput Syst 63:56-75

8. Mahmood K, Naqvi H, Alzahrani BA, Mehmood Z, Irshad A, Chaudhry SA (2018) An ameliorated two-factor anonymous key exchange authentication protocol for mobile client-server environment. Int J Commun Syst 31(18):3814

9. Chen C-M, Huang Y, Wang K-H, Kumari S, Wu M-E (2020) A secure authenticated and key exchange scheme for fog computing. Enterprise Information Systems pp 1-16

10. Chen C-M, Wang K-H, Yeh K-H, Xiang B, Wu T-Y (2019) Attacks and solutions on a three-party passwordbased authenticated key exchange protocol for wireless communications. J Ambient Intell Human Comput 10(8):3133-3142

11. Kumari S, Obaidat M, Wei F et al (2020) Gateway-oriented two-server password authenticated key exchange protocol for unmanned aerial vehicles in mobile edge computing. IET Communications

12. Mahmood K, Arshad J, Chaudhry SA, Kumari S (2019) An enhanced anonymous identity-based key agreement protocol for smart grid advanced metering infrastructure. Int J Commun Syst 32(16):4137

13. Chaudhry SA, Shon T, Al-Turjman F, Alsharif MH (2020) Correcting design flaws: An improved and cloud assisted key agreement scheme in cyber physical systems. Computer Communications

14. Mahmood K, Rehman A, Chaudhary P, Li X, Wu F (2020) Revised anonymous authentication protocol for adaptive client-server infrastructure. Int J Commun Syst 2020:4253

15. Lee W-B, Chang C-C (2000) User identification and key distribution maintaining anonymity for distributed computer networks. Comput Syst Sci Eng 15(4):211-214

16. Wu T-S, Hsu C-L (2004) Efficient user identification scheme with key distribution preserving anonymity for distributed computer networks. Comput Secur 23(2):120-125

17. Tsaur W-J (2001) A flexible user authentication scheme for multi-server internet services. In: International Conference on Networking, pp. 174-183. New York, Springer

18. Li L-H, Lin L-C, Hwang M-S (2001) A remote password authentication scheme for multiserver architecture using neural networks. IEEE Trans Neural Netw 12(6):1498-1504

19. Chang C-C, Lee J-S (2004) An efficient and secure multi-server password authentication scheme using smart cards. In: 2004 International Conference on Cyberworlds, pp. 417-422. IEEE

20. Liao Y-P, Wang S-S (2009) A secure dynamic id based remote user authentication scheme for multi-server environment. Comput Stand Interf 31(1):24-29

21. Hsiang H-C, Shih W-K (2009) Improvement of the secure dynamic id based remote user authentication scheme for multi-server environment. Comput Stand Interf 31(6):1118-1123

22. Lee C-C, Lin T-H, Chang R-X (2011) A secure dynamic id based remote user authentication scheme for multi-server environment using smart cards. Exp Syst Appl 38(11):13863-13870

23. Chen C-T, Lee C-C (2015) A two-factor authentication scheme with anonymity for multi-server environments. Secur Commun Netw 8(8):1608-1625

24. Chang C-C, Lee C-Y (2013) A smart card-based authentication scheme using user identify cryptography. IJ Netw Secur 15(2):139-147

25. Irshad A, Naqvi H, Ashraf Chaudhary S, Usman M, Shafiq M, Mir O, Kanwal A (2018) Cryptanalysis and improvement of a multi-server authenticated key agreement by chen and lee's scheme. Inform Technol Contr 47(3):431-446

26. Kumari S, Das AK, Li X, Wu F, Khan MK, Jiang Q, Islam SH (2018) A provably secure biometrics-based authenticated key agreement scheme for multi-server environments. Multim Tools Appl 77(2):2359-2389 
27. Li X, Peng J, Obaidat MS, Wu F, Khan MK, Chen C (2020) A secure three-factor user authentication protocol with forward secrecy for wireless medical sensor network systems. IEEE Syst J 14(1):39-50

28. Li X, Wu F, Kumari S, Xu L, Sangaiah AK, Choo K-KR (2019) A provably secure and anonymous message authentication scheme for smart grids. J Parallel Distrib Comput 132:242-249

29. Altaf I, Arslan Akram M, Mahmood K, Kumari S, Xiong H, Khurram Khan M (2020) A novel authentication and key-agreement scheme for satellite communication network. Trans Emerg Telecommun Technol. https://doi. org/10.1002/ett.3894

30. Arshad R, Ikram N (2013) Elliptic curve cryptography based mutual authentication scheme for session initiation protocol. Multim Tools Appl 66(2):165-178

31. Amin R, Biswas G (2015) Design and analysis of bilinear pairing based mutual authentication and key agreement protocol usable in multi-server environment. Wireless Pers Commun 84(1):439-462

32. Amin R, Islam SH, Biswas G, Khan MK, Leng L, Kumar N (2016) Design of an anonymity-preserving three-factor authenticated key exchange protocol for wireless sensor networks. Comput Netw 101:42-62

33. Irshad A, Chaudhry SA, Xie Q, Li X, Farash MS, Kumari S, Wu F (2018) An enhanced and provably secure chaotic mapbased authenticated key agreement in multi-server architecture. Arab J Sci Eng 43(2):811-828

34. Ravanbakhsh N, Nazari M (2018) An efficient improvement remote user mutual authentication and session key agreement scheme for e-health care systems. Multim Tools Appl 77(1):55-88

35. Islam SH, Obaidat MS, Amin R (2016) An anonymous and provably secure authentication scheme for mobile user. Int J Commun Syst 29(9):1529-1544

36. Tsai J-L (2008) Efficient multi-server authentication scheme based on one-way hash function without verification table. Comput Secur 27(3-4):115-121

37. Shamshad S, Mahmood K, Kumari S (2020) Comments on 'a multi-factor user authentication and key agreement protocol based on bilinear pairing for the internet of things.' Wireless Personal Communications pp 1-4

38. Amin R, Islam SH, Biswas G, Khan MK, Kumar N (2018) A robust and anonymous patient monitoring system using wireless medical sensor networks. Fut Gen Comput Syst 80:483-495

39. Kalra S, Sood SK (2015) Secure authentication scheme for iot and cloud servers. Perv Mob Comput 24:210-223

40. Alshahrani M, Traore I (2019) Secure mutual authentication and automated access control for iot smart home using cumulative keyed-hash chain. J inform Secur Appl 45:156-175

41. Potlapally NR, Ravi S, Raghunathan A, Jha NK (2006) A study of the energy consumption characteristics of cryptographic algorithms and security protocols. IEEE Trans Mob Comput 5(2):128-143

\section{Publisher's Note}

Springer Nature remains neutral with regard to jurisdictional claims in published maps and institutional affiliations.

\section{Submit your manuscript to a SpringerOpen ${ }^{\circ}$ journal and benefit from:}

- Convenient online submission

- Rigorous peer review

- Open access: articles freely available online

- High visibility within the field

- Retaining the copyright to your article

Submit your next manuscript at $\boldsymbol{\nabla}$ springeropen.com 